9 Whitehouse AM, Duncan JM. Ephedrine psychosis rediscovered. Br J Psychiatry 1987;150:258-61.

10 Pugh CR, Howie SM. Dependence on pseudoephedrine. $\mathrm{Br}$ Psychiatry 1986;149:798.

11 Jelley TM. Phensedyl abuse, Br $\mathcal{F}$ Psychiatry 1987;151:418-9.

12 Lambert T. Paranoid psychoses after abuse of proprietary cold remedies. Br f Psychiatry (in press).

\section{Refeeding hypophosphataemia in anorexia nervosa and alcoholism}

SIR,-The article by Dr Allan D Cumming and colleagues on refeeding hypophosphataemia (22 August, p 490) omits a number of salient points relating to the development of convulsions in alcoholics with complex biochemical disturbances. Several combinations of the abnormalities de scribed might account for the symptoms observed.

Magnesium depletion, in particular, is well known to be a common cause of convulsions in such patients. ${ }^{1}$ In addition, magnesium deficiency induces hypokalaemia and hypocalcaemia, as well as hypomagnesaemia. ${ }^{2}$ In the alcoholic patient described the potential contributions of other ionic imbalances, notably hypokalaemia $(3.2 \mathrm{mmol} / \mathrm{l})$ and hypomagnesaemia $(0.36 \mathrm{mmol} / \mathrm{l})$, are ignored. This is surprising in view of the fact that this patient required "separate infusions of calcium magnesium, and potassium" before recovery.

Though we should not ignore the potential importance of refeeding hypophosphataemia, the contribution of hypophosphataemia itself to the symptoms described remains unclear.

M F RYAN H PANDOV

Department of Clinical Chemistry,

East Birmingham Hospital,

Birmingham B9 5ST

1 Berkelhammer C, Bear RA. A clinical approach to commo electrolyte disorders. Hypomagnesaemia. Can Med Assoc 1985;132:360-8.

2 Brenton DP, Gordon TE. Magnesium. Br f Hosp Med 1984;32. $60-9$

AUTHORS' REPLY,-Drs Ryan and Pandov seem to have misunderstood the purpose of our article which was to emphasise the importance of rapid changes in the plasma phosphate concentration during the refeeding of malnourished patients. Their comments illustrate the confusion that commonly arises between the effects of electrolyte disturbances during chronic nutritional deficiency and acute electrolyte changes after treatment is started. The biochemical variables of our second patient on admission typified those of severe alcoholism: hyponatraemia, hypokalaemia, hypomagnesaemia, and moderate hypophosphataemia. These changes were probably of long standing, and the patient was asymptomatic. Subsequent clinical deterioration after feeding was associated with a decline of plasma phosphate concentration from 0.30 to $0.10 \mathrm{mmol} / \mathrm{l}$, a value indicating severe hypophosphataemia and likely to cause symptoms. Plasma magnesium concentration did not change. As stated in our report, plasma calcium and magnesium concentrations fell during intravenous treatment with phosphate; these are recognised effects of intravenous phosphate administration and were treated as necessary. ${ }^{2}$

Clinically, electrolyte disturbances rarely occur in isolation, and speculation about the relative contribution of each abnormality to the patient's clinical state is seldom helpful. We were careful not to suggest an exclusive causal relation between symptoms and hypophosphataemia, and indeed we commented in the discussion that hypokalaemia, hypomagnesaemia, and acid base disturbance may have contributed. Magnesium depletion is certainly a common feature of chronic alcoholism. We suspect, however, that hypo- magnesaemia was not the primary cause of symptoms in either patient. Neither showed the most common symptoms associated with hypo magnesaemia - paraesthesia, cramp, and tetany. Magnesium deficiency usually induces hypocalcaemia, as Drs Ryan and Pandov comment, ${ }^{4}$ but plasma calcium concentration was normal in both cases. Hypophosphataemia was profound $(<20 \%$ of normal) at the onset of symptoms, with comparatively less depression of plasma magnesium and potassium concentrations. Experimentally, selective phosphate depletion causes magnesuria and hypomagnesaemia ${ }^{35}$; magnesium depletion may cause intracellular phosphate depletion, but the plasma phosphate concentration remains normal.

In relation to the opening sentence by Drs Ryan and Pandov, it should be noted that at no point in our paper do we refer to convulsions in an alcoholic patient. The patient in case 1 suffered from anorexia nervosa alone, and convulsions were not bserved in case 2

We suspect that the clinical importance of changes in the plasma phosphate concentration is not always recognised. We would emphasise that in malnourished patients an "admission blood" will not detect progressive hypophosphataemia during refeeding, and we recommend continued biochemical monitoring until the patient is clinically stable.

Allan D Cumming JACQUELYN R FARQHAR IAN A D BOUCHIER

Edinburgh University Department of Medicine,

Royal Infirmary, Edinburgh EH3 9YW

1 Berkelhammer C, Bear RA. A clinical approach to common electrolyte problems: 3 . Hypophosphatemia. Can Med Assoc $f$ 1984;130:17-23.

Kurokawa K, Levine BS, Lee DBN, Massry SG. Physiology of phosphorus metabolism and pathophysiology of hypophosphatemia and hyperphosphatemia. In: Areiff AI, De Fronzo RA, eds. Fluid, electrolyte and acid base disorders. New York: Churchill Livingstone, 1986.

3 Brenton DP, Gordon TE. Magnesium. Br $\mathcal{F}$ Hosp Med 1984;32: 60-9.

Cronin RE, Knochel JP. Magnesium deficiency. Ado Intern Med 1983;28:509-33.

Massry SG. The clinical syndrome of phosphate depletion. Ado Exp Med Biol 1978;103:301-12.

False positive results of tests for syphilis and outcome of pregnancy

SIR,-Dr J G Thornton and coworkers (8 August, p 354) conclude that pregnant women with a false positive result in one of three non-specific serological tests for syphilis had an increaed rate of pregnancy loss during the first and second trimesters. We are, however, uncertain whether this association is valid.

Only 39 of 71 patients were retested and confirmed as being seropositive, and therefore technical false positive reactions were not excluded in the remaining women. "Biological false positive" reactions may be transient or chronic, the latter being present for more than six months. Acute false positive reactions may be associated with infection-for example, herpes simplex virusparticularly when fever is present. Chronic reactions have been reported in intravenous drug users. These maternal conditions may contribute to spontaneous abortion and increased fetal loss. False positive reactions are recognised as a seroogical abnormality in autoimmune disease, bein more common in women and often predating the clinical manifestations by many years.

Though retrospective matching of the cases was closely related to obstetric performance, there were no reported attempts at matching for ethnic background or social class, which is particularly surprising with regard to ethnic background in view of the higher prevalence of systemic lupus erythematosus in negro women. ${ }^{2}$ We also do not know whether metabolic conditions, such as maternal diabetes mellitus, were taken into account in the study.

Sensitive though these non-specific treponemal tests are, we would argue that they remain an inaccurate screening method for detecting maternal autoimmune disease. The results may be confounded by the exogenous or endogenous factors noted above, just as spontaneous abortions and fetal loss have a multifactorial aetiology.

R LAU

G E FORSTER

The London Hospital (Whitechapel),

London E1 1BB

King A, Nicol C, Rodin P. Venereal diseases. 4th ed. London: Bailliere Tindall, 1980.

Fessel WJ. Systemic lupus erythematosus in the community. Arch Intern Med 1974;134:1027.

Prognosis of subsequent pregnancies after recurrent spontaneous abortion in first trimester

SIR,-The message of the article by Drs Willem Vlaanderen and Pieter E Treffers (11 July, p 92) is that 17 of 21 patients had subsequently successful pregnancies after recurrent spontaneous abortion and that therefore extensive investigation and treatment are unnecessary. Our experience, based on the assessment of over 200 women with a history of recurrent abortion, is quite different.

We agree that the loss of a vital healthy embryo is rare and that abortion in the first trimester is usually preceded by death of the embryo. In our series of 413 first trimester abortions in women who habitually aborted (out of 800 that could be assessed in 186 women), only 31 were abortions of live embryos, 382 being missed abortions. It is unjustified, however, to claim that most aborted fetuses show chromosomal anomalies. Less than $20 \%$ of aborted fetuses may be successfully karyotyped. ${ }^{12}$ Live tissue is necessary for karyotyping, and the necrotic trophoblast obtained from a missed abortion does not usually grow sufficiently in culture to allow karyotyping. It is mere supposition that these abortions have a chromosomal or ovular basis.

Drs Vlaanderen and Treffers conclude that extensive examination is seldom helpful. We agree, but we disagree that recurrent embryonic death in women with a history of recurrent abortion must be regarded as inevitable; it is precisely this type of pregnancy that responds to immunotherapy. Drs Vlaanderen and Treffers quote the results of Mowbray et al: 17 successful pregnancies out of $22 .{ }^{3} \mathrm{We}$ also consistently find that $80 \%$ of subsequent pregnancies progress normally after immunisation. Drs Vlaanderen and Treffers report that 17 of 21 women subsequently had successful pregnancies. Our experience, however, is completely different. Only seven out of 28 women with a history of recurrent abortion who did not receive treatment subsequently had successful pregnancies.

Drs Vlaanderen and Treffers describe patients who had had three or more abortions. Do their suggestions, however, of little investigation and no treatment (apart from reassurance and encouragement) pertain to women who have had five or more abortions?

Our results in women who had had five or more missed abortions in the first trimester (abortions of live embryos are exluded) show that only three of 14 subsequent pregnancies progressed normally, as opposed to 18 of 24 after paternal leucocyte 
immunisation. This difference was significant ( $p=$ $<0.005, \chi^{2}$ analysis). While it is assumed that half of those women might have a live birth after numerous additional abortions, these abortions may be prevented by immunotherapy. Moreover, women who have had one spontaneous abortion who have their immune response altered by immunotherapy seem to have second subsequently successful pregnancies. (This has occurred in all five patients who have become pregnant a second time in our series.) Though such women have spontaneously successful pregnancies, however, they seem to revert to their tendency to abort afterwards. Nine patients in our series had two or more abortions after a spontaneously successful pregnancy. Only one patient had two subsequently successful pregnancies after one spontaneous abortion.

We therefore consider immunotherapy to be a most effective form of treatment for women who have had missed abortions, and the results of Drs Vlaanderen and Treffers do not convince us otherwise.

V TODER

D M SERR

S MASHIACF

Department of Obstetrics and Gynaecology, Sheba Medical Centre, Tel-Hashomer, and Department of Embryology and Teratology,

Tel-Aviv University, Israel

1 Ornoy A, Salomon J, Ben Zur Z, Kohn G. Placental findings in spontaneous abortions and stillbirths. Teratology 1981;21:243 52.

2 Kardon NB, Davis JG, Berger A, Broekman A. Incidence of chromosomal rearrangement in couples with reproductive loss. Hum Genet 1980;53:161-4.

3 Mowbray JF, Gibbings CR, Underwood JL, Liddel H, Reginald RW, Beard RW. Controlled trial of treatment of recurren spontaneous abortions by immunisation with paternal cells. Lancet 1985; i:941-3.

\section{Emotional distress in junior doctors}

SIR,-Ms Jenny Firth-Cozens (29 August, p 533) suggests that problems of stress in clinical work should be dealt with in the undergraduate medical curriculum. ${ }^{1}$ I agree, but I would add that we still have much to learn about the most appropriate way of doing this.

Some preliminary observations from Bristol's new undergraduate management teaching may be of interest. This forms part of our clinical community medicine course in the fourth year and is perhaps an example of the "innovative approach to management education" being sought by the National Health Service Training Authority. ${ }^{2}$ Doctors are an expensive commodity; it makes good sense to help them to maintain and maximise their performance in delivering health care.

The focus of our approach is the clinical ward team, as experienced by house officers. Through this teaching I have become aware of the mismatch between our medical training, which encourages extreme competitiveness between individuals, and the requirements of good medical care, which demands a high level of teamwork. As a corollary, the type of training that we provide may inhibit rather than encourage many students from seeking support when under stress. In a series of voluntary workshops on "Coping with the demands of becoming a doctor: stress and satisfaction" students have been surprised to discover that most of their peers are also experiencing feelings of inadequacy and uncertainty about the nature of their task. They are often relieved to find that they are not, after all, peculiar. Students also speak of the scarcity of opportunities in the medical course for admitting such emotions.

As part of the core teaching on teamwork senior nursing and medical students examine potential areas of cooperation and conflict hetween house officers and staff nurses. This has shown considerable misunderstanding and negative feelings between the two groups, with examples of how such feelings may increase the stress on all concerned. For instance, doctors who fail to communicate adequately with the nursing staff about patient care or are insensitive to the pressures of nurses' work routines increase their risk of being woken at night.

Evaluation of this and our other teaching on teamwork shows that it is popular and there is demand for more. As a result of these workshops, students have developed together some positive "action plans," describing what they would like to do, as staff nurses and house officers, to improve working relationships and provide each other with more support. It is too soon, however, to determine whether this results in changed behaviour. We would be interested to hear from others who are exploring these issues at the undergraduate and house officer levels.

Department of Epidemiology and Community Medicine,

Canynge Hall,

Whiteladies Road,

Bristol BS8 2PR

1 Firth J. Levels and sources of stress in medical students. BrMed J 1986;292:1177-80.

2 National Health Service Training Authority. Developing the role of doctors in the management of the National Health Service. A of doctors in the management of the National
discussion document. London: NTA, 1986:23.

SIR,-The paper by Ms Jenny Firth-Cozens on emotional distress in junior house officers (29 August, p 533) is important and will, we hope, not go unnoticed either by consultants or by employing authorities. A similar but less detailed study was undertaken in the South Western region three years ago (precipitated by the suicide of a junior doctor) and produced similar results. Without any financial incentive, the rate of response to our questionnaire was only $38 \%$, but this nevertheless produced 397 responses. There was a high incidence of crises related to workload, the stress of the job, and worries about career prospects.

Many replies showed considerable cynicism and bitterness about the medical establishment and the lack of sympathy with the problems of sheer overwork experienced by junior doctors. Many thought that the present system of obtaining help was unsatisfactory.

Our reaction to our studies was to encourage each district to set up a counselling system for junior doctors, either within their own specialty through tutors and regional advisers or, if the problem was not specialty based, through the general practice clinical tutor, with the regional postgraduate dean's office being available to all We emphasised that these services should be adequately advertised and freely available. It also became clear that many junior doctors, because of their frequent changes of place of employment never registered with a general practitioner, and we have requested that employing authorities make this a condition of employment so that, in common with the rest of the community, junior doctors at least have the basic important support of a family doctor.

Most of the other points made by Ms FirthCozens were echoed by our own studies. We believe that the problem is considerable and one that should be seen as a major responsibility for a caring profession.

C J Burns-Cox K KEARSEY

Medical Postgraduate Department, J B M ROBERTS

University of Bristol,

Bristol BS8 2PR

\section{Is the tube in the trachea?}

SIR,-Over the past few weeks I have been looking into various methods used to place endotracheal tubes and cuffs in the trachea correctly and confirm their positions. Drs Peter A Coe and R M Towey (19 September, p 723) rightly point out that the most reliable method available to check the position of a tube after non-visualised, difficult intubation is with an intubating fibrescope. Though this test is simple and extremely easy to perform, I would disagree with them that no previous experience is needed to identify the mucous membrane overlying the tracheal rings and the carina from the mucous membrane of the oesophagus. I would therefore suggest that during their training anaesthetists should routinely use a fibreoptic bronchoscope through the endotracheal tube so that they learn to recognise the tracheal rings and the carina.

A new process has been developed, based on the transcutaneous detection of a specially designed tracheal tube that contains a magnetic marker (Track Mate intubation system, McCormick Laboratories Inc). The sensing probe transcutaneously detects the position of the magnetic marker by producing light and sound signals, the intensity of which correlates with the proximity of the tube marker to the probe.

Patients do not die from "failure to intubate."1 They die from failure to stop trying to intubate or from undiagnosed oesophageal intubation. It is therefore important to remove the tube and ventilate the patient with a mask when there is doubt about the correct placement of the tube associated with clinical deterioration.

Department of Anaesthetics,

S MEHTA Burnley General Hospital,

Burnley BB10 2PQ

1 Scott DB. Endotracheal intubation: friend or foe. $\mathrm{Br} \mathrm{Med} \mathrm{J}$ 1986;292:157-8.

SIR,-While direct visualisation of the trachea through a flexible endoscope passed through the tube as suggested by Drs P A Coe and R M Towey (19 September, p 723), seems attractive, we would caution against its routine use because we believe that corners will be cut when the instrument requires sterilisation. Sterilisation requires at least 30 minutes' immersion in $2 \%$ gluteraldehyde solution, ${ }^{1}$ and more than one bronchoscope for each theatre suite will almost certainly be required, making this a potentially expensive exercise.

R A S FRATER P G P LAWLER

South Cleveland Hospital,

Cleveland TS4 3BW

I Department of Health and Social Security. Decontamination of equipment, linen or other surfaces contaminated with hepatitis $B$ or human immunodeficiency vinus. London: HMSO, 1987. (Health Notice HN(87)1.)

\section{Acyclovir for shingles}

SIR,-The Wellcome Foundation Ltd advertises a course of acyclovir for shingles at a basic cost of £119.

Nobody doubts the need to treat a high risk attack-for example, ophthalmic shingles or the Ramsey-Hunt syndrome-with all possible means, but surely it is misleading to imply that we should use it for every attack.

Of the 80 patients presenting to me with shingles in general practice over nine years, 25 were aged less than 50 , and none of these were suffering from a severe attack. All that was needed was reassur- 